Kutzelmann, Sabine; Theinert, Kerstin

Miteinander sind alle Stars - Die Bedeutung des kooperativen Lernens im Mehrsprachigen Lesetheater

Mehrsprachiges Lesetheater. Handbuch zu Theorie und Praxis. Opladen; Berlin; Toronto : Verlag Barbara Budrich 2017, S. 120-131

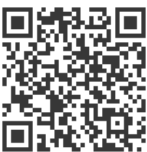

Quellenangabe/ Reference:

Kutzelmann, Sabine; Theinert, Kerstin: Miteinander sind alle Stars - Die Bedeutung des kooperativen Lernens im Mehrsprachigen Lesetheater - In: Mehrsprachiges Lesetheater. Handbuch zu Theorie und Praxis. Opladen; Berlin; Toronto : Verlag Barbara Budrich 2017, S. 120-131 - URN: urn:nbn:de:0111-pedocs-216703 - DOI: 10.25656/01:21670

https://nbn-resolving.org/urn:nbn:de:0111-pedocs-216703

https://doi.org/10.25656/01:21670

in Kooperation mit / in cooperation with:

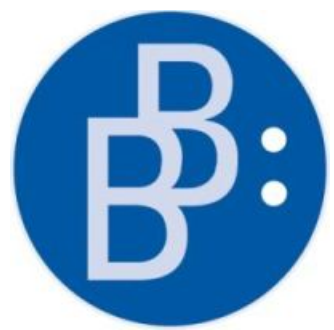

https://www.budrich.de

\section{Nutzungsbedingungen}

Gewährt wird ein nicht exklusives, nicht übertragbares, persönliches und beschränktes Recht auf Nutzung dieses Dokuments. Dieses Dokument ist ausschließlich für den persönlichen, nicht-kommerziellen Gebrauch bestimmt. Die Nutzung stellt keine Übertragung des Eigentumsrechts an diesem Dokument dar und gilt vorbehaltlich der folgenden Einschränkungen: Auf sämtlichen Kopien dieses Dokuments müssen alle Urheberrechtshinweise und sonstigen Hinweise auf gesetzlichen Schutz beibehalten werden. Sie dürfen dieses Dokument nicht in irgendeiner Weise abändern, noch dürfen Sie dieses Dokument für öffentliche oder kommerzielle Zwecke vervielfältigen, öffentlich ausstellen, aufführen, vertreiben oder anderweitig nutzen.

Mit der Verwendung dieses Dokuments erkennen Sie die Nutzungsbedingungen an.

\section{Kontakt / Contact:}

peDOCS

DIPF | Leibniz-Institut für Bildungsforschung und Bildungsinformation Informationszentrum (IZ) Bildung

E-Mail: pedocs@dipf.de

Internet: www.pedocs.de

\section{Terms of use}

We grant a non-exclusive, non-transferable, individual and limited right to using this document.

This document is solely intended for your personal, non-commercial use. Use of this document does not include any transfer of property rights and it is conditional to the following limitations: All of the copies of this documents must retain all copyright information and other information regarding legal protection. You are not allowed to alter this document in any way, to copy it for public or commercial purposes, to exhibit the document in public, to perform, distribute or otherwise use the document in public.

By using this particular document, you accept the above-stated conditions of use. 
Sabine Kutzelmann Ute Massler Klaus Peter Kristina Götz Angelika Ilg (Hrsg.)

\section{Mehrsprachiges Lesetheater}

Handbuch zu Theorie und Praxis 
Mehrsprachiges Lesetheater 

Sabine Kutzelmann • Ute Massler • Klaus Peter • Kristina Götz • Angelika Ilg (Hrsg.)

\section{Mehrsprachiges Lesetheater}

Handbuch zu Theorie und Praxis

Verlag Barbara Budrich

Opladen • Berlin • Toronto 2017 
Bibliografische Information der Deutschen Nationalbibliothek

Die Deutsche Nationalbibliothek verzeichnet diese Publikation in der Deutschen Nationalbibliografie; detaillierte bibliografische Daten sind im Internet über http://dnb.d-nb.de abrufbar.

Gefördert durch

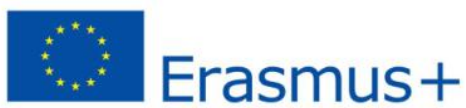

Schulbildung

Dieses Projekt wurde mit Unterstützung der Europäischen Kommission finanziert. Die

Verantwortung für den Inhalt dieser Veröffentlichung (Mitteilung) trägt allein der Verfasser; die Kommission haftet nicht für die weitere Verwendung der darin enthaltenen Angaben.

This project has been funded with support from the European Commission. This publication reflects the views only of the author, and the Commission cannot be held responsible for any use which may be made of the information contained therein.

Gedruckt auf säurefreiem und alterungsbeständigem Papier.

Alle Rechte vorbehalten.

C 2017 Verlag Barbara Budrich, Opladen, Berlin \& Toronto

www.budrich-verlag.de

$$
\text { ISBN 978-3-8474-2021-4 }
$$

eISBN 978-3-8474-1109-3

Das Werk einschließlich aller seiner Teile ist urheberrechtlich geschützt. Jede Verwertung außerhalb der engen Grenzen des Urheberrechtsgesetzes ist ohne Zustimmung des Verlages unzulässig und strafbar. Das gilt insbesondere für Vervielfältigungen, Übersetzungen, Mikroverfilmungen und die Einspeicherung und Verarbeitung in elektronischen Systemen.

Umschlaggestaltung: Bettina Lehfeldt, Kleinmachnow - www.lehfeldtgraphic.de

Lektorat und Satz: Ulrike Weingärtner, Gründau

Titelbildnachweis: Stillaufnahme aus dem MELT Film „LOUD, DEUTLICH, LENTEMENT: Mit

Mehrsprachigem Lesetheater Sprachgrenzen überwinden“ (s. Projektwebsite: http://melt-multilingualreaders-theatre.eu/ )

Druck: paper \& tinta, Warschau

Printed in Europe 


\section{Inhalt}

Das Projekt Mehrsprachiges Lesetheater: Ausgangslage, Zielsetzung

und praxisrelevante Ergebnisse der Entwicklung .................

Sabine Kutzelmann, Ute Massler und Robert Hendel

\section{Teil A}

\section{Forschungsmethodik}

1 Design-Based Research: Eine Forschungsstrategie zur Entwicklung nachhaltiger Innovationen in der schulischen Praxis . . . . . . . . . . . . Sabine Kutzelmann und Kristina Götz

2 Die schulischen Erprobungskontexte des MELT-Projekts . . . . . . . . . . . 32 Kristina Götz, Robert Hendel, Sabine Kutzelmann und Klaus Peter

3 Mehrsprachiges Lesetheater: Ergebnisse zur Akzeptanz dieser

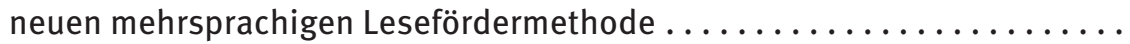
Kristina Götz, Sabine Kutzelmann und Ute Massler

Teil B

Theoretische Grundlagen des

Entwicklungs- und Forschungsprozesses

1 Leseflüssigkeit und Lesemotivation: Die beiden Förderdimensionen des Mehrsprachigen Lesetheaters . . . . . . . . . . . . . . . Kristina Götz und Sabine Kutzelmann

2 Das Unterrichtsdesign Mehrsprachiges Lesetheater aus linguistischer Sicht.

Klaus Peter und Dominik Unterthiner

3 Vom Readers' Theatre über interlinguale Literatur zum Mehrsprachigen Lesetheater: Die didaktisch-methodischen Leitlinien der Gestaltung im Kontext der Mehrsprachigkeitsdidaktik ................. Ute Massler und Sabine Kutzelmann

\section{Teil C}

\section{Das didaktisch-methodische Design:}

\section{Theoretische Grundlagen und Anleitung für die Praxis}

1 Mehrsprachige Lesetheaterstücke: Konzeption der Lehr-Lern-Materialien . . . Angelika Ilg, Franz Ludescher und Seraina Paul

2 Mehrsprachiges Vorlesen durch die Lehrperson als Design-Komponente des Mehrsprachigen Lesetheaters . . . . . . . . . . . . . . . . .

Ute Massler und Kerstin Theinert 
3 Miteinander sind alle Stars - Die Bedeutung des kooperativen Lernens

im Mehrsprachigen Lesetheater.

120

Sabine Kutzelmann und Kerstin Theinert

4 Literarisches Lernen als Design-Komponente des

Mehrsprachigen Lesetheaters . . . . . . . . . . . . . . . . .

Angelika Ilg und Kerstin Theinert

5 Die zentralen Lehr-Lern-Prozesse des Mehrsprachigen Lesetheaters:

Eine Anleitung für die Praxis

Sabine Kutzelmann, Ute Massler, Klaus Peter unter Mitarbeit von

Anne-Marie Antony, Stefanie Arnold, Kurt Benedikt, Andrea Büchel,

Stefanie Blodnig, Tessy Gollère, Robert Hendel und Christian Mertens

\section{Teil D}

\section{Curriculum Lehrerfortbildung}

1 Professionswissen und Einstellungen von Sprachlehrpersonen zur

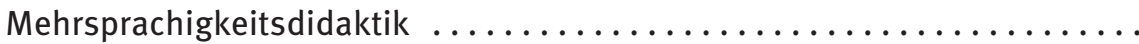
Klaus Peter und Dominik Unterthiner

2 Sprach- und Fachgrenzen überwinden: Die didaktische und strategische Konzeption des Fortbildungscurriculums zur Lesefördermethode

Mehrsprachiges Lesetheater ......................... 168

Sabine Kutzelmann und Ute Massler

Anhang 1) Anleitung zur Dramatisierung von mehrsprachigen Lesetheatern am Beispiel von Bend it like Beckham . . . . . . . . . . . . . . . . 181 Anhang 2) Lesetheater Heidi . . . . . . . . . . . . . . . . . . . . . . 185

Anhang 3) Lesetheater Luina - Die Seejungfrau vom Bodensee . . . . . . . . . . . 189

Anhang 4) Lesetheater Nasreddin Hodscha . . . . . . . . . . . . . . . . . . . . 191

Anhang 5) Lesetheater Dr Jekyll and Mr Hyde . . . . . . . . . . . . . . . . 195

Anhang 6) Toolbox: Dramapädagogik ...................... 196

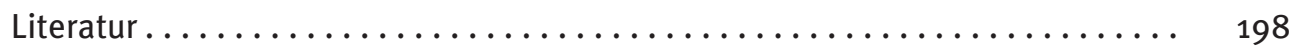

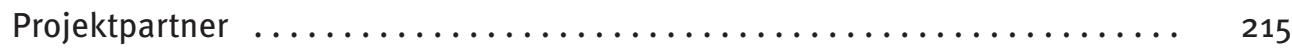

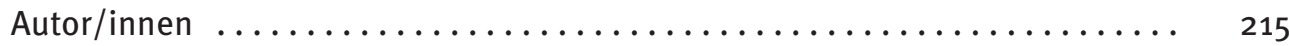




\title{
$3 \quad$ Miteinander sind alle Stars - Die Bedeutung des kooperativen Lernens im Mehrsprachigen Lesetheater
}

\author{
Sabine Kutzelmann und Kerstin Theinert
}

Eine Besonderheit des didaktisch-methodischen Designs Mehrsprachiges Lesetheater besteht darin, dass der mehrteilige und sich über drei bis fünf Unterrichtsstunden erstreckende Lehr- und Lernprozess überwiegend als kooperatives Lernen organisiert ist. Nach den beiden lehrerzentrierten Schritten der Einführung des Lesetheaters und des Vorlesens durch die Lehrperson, die zu Beginn der Arbeit mit einem mehrsprachigen Lesetheaterstück im Klassenverband stattfinden, wird die Klasse in Kleingruppen unterteilt, um arbeitsteilig jeweils eine Szene für die den Lernprozess abschließende Aufführung im Klassenverband vorzubereiten. Eine solche gruppenbasierte Unterrichtsorganisation führt allerdings nicht zwingend zu Kooperation, darum galt es im Entwicklungs- und Forschungsprozess zu dieser Design-Komponente, Fragestellungen wie den folgenden nachzugehen und praktisch umsetzbare Lösungen zu finden: Wie sollen in diesem arbeitsteiligen Prozess die Schüler/innen konkret interagieren? Welche Teilaufgaben müssen gestellt werden, damit in den Gruppen die Vorlesegestaltung einer Szene sinnvoll miteinander erarbeitet wird? Welche Zielsetzungen werden bei der jeweiligen Teilaufgabe verfolgt? Welche möglichen Herausforderungen und Schwierigkeiten sind bei der Umsetzung in die Praxis zu berücksichtigen?

Vor dem Hintergrund dieser Fragestellungen beschäftigt sich das folgende Kapitel mit den konzeptuell-theoretischen Grundlagen, die für den Entwicklungs- und Forschungsprozess dieser Design-Komponente maßgeblich waren. Zunächst erfolgt die Darstellung einiger wesentlicher Merkmale des kooperativen Lernens, die auch in Hinblick auf die praktische Realisierung des kooperativen Lernens einen hohen Stellenwert besitzen (3.1). Aufgrund der großen Bedeutung der Lehrperson beim kooperativen Lernen wird in einem zweiten Schritt auf ihre Rolle und ihre Aufgaben insbesondere bei der Einführung der Arbeit mit einem mehrsprachigen Lesetheaterstück eingegangen (3.2). Mehrsprachiges Lesetheater soll als Lesefördermethode in erster Linie dazu beitragen, dass die Schüler/innen flüssiger und motivierter lesen. Trotz dieses Förderfokus darf aber davon ausgegangen werden, dass aufgrund des kooperativen Lernens auch die sozialen Kompetenzen der Schüler/innen gefördert werden. Darum werden abschließend relevante Studienergebnisse zur Wirksamkeit von kooperativen Lernformen in Bezug auf die im Design angestrebten sozialen und kommunikativen Lernziele ausblickend diskutiert (3.3).

\subsection{Merkmale des kooperativen Lernens}

Unter kooperativem Lernen versteht man im weitesten Sinne eine Interaktionsform, bei der zwei oder mehr Personen gemeinsam das Ziel verfolgen, etwas zu lernen. Da- 
bei erwerben die beteiligten Personen in wechselseitigem Austausch Kenntnisse und Fertigkeiten. Idealerweise nehmen alle Gruppenmitglieder gleichberechtigt am Lerngeschehen teil und tragen gemeinsam Verantwortung (vgl. Huber 1999, 2011; Konrad/ Traub 2001, 2008). Der Begriff „kooperatives Lernen“ wird darum eng mit dem komplementären Konzept des selbstgesteuerten Lernens in Verbindung gebracht (vgl. Bonnet/ Küppers 2011: 37).

Um das Wesentliche des kooperativen Lernens zu erfassen und von anderen Organisationsformen des Lernens (z. B. kompetitive oder individualistische Formen, vgl. Johnson/Johnson 1999: 6ff.; Borsch 2015: 16ff.) abzugrenzen, beschreiben Johnson/ Johnson (1999: 69ff.) basic elements of cooperative learning; diese werden im deutschsprachigen Raum von verschiedenen Autor/innen aufgegriffen und als zentrale Merkmale kooperativen Lernens dargestellt (vgl. z. B. Bonnet/Küppers 2011: 37, Borsch 2015: 27ff., Konrad/Traub 2008: 140): (1) positive Interdependenz (2) individuelle Verantwortlichkeit, (3) hilfreiche Face-to-Face-Interaktion, (4) Feedback, (5) angemessene Nutzung kooperativer Fertigkeiten und (6) konstruktive Unterstützung und Reflexion der Gruppenprozesse.

Im Folgenden wird erläutert, wie diese Basiselemente kooperativen Lernens bei der Konzeption des Lehr-Lern-Prozesses im Mehrsprachigen Lesetheater berücksichtigt wurden:

(1) Positive Interdependenz: Die mehrsprachigen Lesetheaterstücke, die im Projekt als Lernmaterialien eingesetzt wurden, setzen sich aus mehreren Szenen zusammen, die innerhalb einer Klasse auf Untergruppen verteilt werden. Für eine Gruppe besteht die Zielsetzung darin, ihre Szene so zu bearbeiten und zu üben, dass sie am Ende des Erarbeitungsprozesses, im Klassenverband ausdrucksstark vorgelesen werden kann. Um dieses gemeinsame Ziel zu erreichen, müssen die in der Szene vorkommenden Erzähler- und Figurenrollen auf die einzelnen Mitglieder einer Gruppe verteilt werden, die sich dann gezielt auf das gestaltende Vorlesen „,ihrer“ Rollen spezialisieren. Diese Strukturierung des Lernprozesses wie auch die Zielsetzung bedingen, dass die Schüler/innen einer Arbeitsgruppe in einem positiven Sinne voneinander abhängig sind, weil man nur gemeinsam erfolgreich sein kann. Um zu einer Gesamtdeutung der vorzulesenden Szene zu gelangen, müssen sie ihre individuelle Vorlesegestaltung mit den anderen Mitgliedern der Gruppe abstimmen und Ideen zur Inszenierung des Textes austauschen. Die Gruppenmitglieder müssen also kooperieren, weil sie aufeinander angewiesen sind. Löst jemand seine Teilaufgabe nicht angemessen, indem beispielsweise die eigene Leserolle nur oberflächlich geübt wird oder sich jemand als einziger vor den anderen als der „Star“ hervortun will, kann die Gruppe als Ganzes bei der Aufführung nicht überzeugend vor dem Publikum auftreten. Diese positive Interdependenz kann noch verstärkt werden, wenn die Gruppe eine eigene Identität entwickelt (z. B. mit Namen von Figuren aus einem Lesetheaterstück oder verschiedenfarbigen Shirts). Konkurrieren Schüler/innen um Leistungen wie beispielsweise bei einem Vorlese-Wettbewerb, entsteht eine negative Interdependenz, denn das Ziel besteht darin, sich mit anderen zu messen und besser als andere Schüler/innen zu lesen, um im Wettbewerb zu siegen.

Die Aufgabenstellung gliedert sich in mehrere Teilaufgaben, die von den vier bis sechs Arbeitsgruppen selbstständig bearbeitet werden sollen (s. Tab. $1 \mathrm{im}$ folgenden Abschnitt 3.2): vom Kennenlernen der einzelnen Szenen durch selbstständige Lektü- 
re und der Rollenverteilung über das Erarbeiten einer Inhaltsangabe der Lesetheaterszene und einer Kurzpräsentation, weiter zum intensiven Üben der Leserollen durch wiederholtes halblautes Lesen in Einzel-, Partner- oder Gruppenarbeit bis hin zur Generalprobe und der den Lernprozess abschließenden Aufführung des mehrsprachigen Lesetheaters im Klassenverband. Diese Gliederung in Teilaufgaben macht deutlich, dass die Schüler/innen nicht durchgängig mit den anderen Gruppenmitgliedern zusammenarbeiten, sondern es Phasen gibt, in denen sie auch für sich allein oder zu zweit arbeiten. Diese Phasen sind zentral, damit sie ihre individuelle Verantwortung wahrnehmen können.

(2) Individuelle Verantwortlichkeit: Für die Gestaltung der eigenen Rolle ist im Wesentlichen jede/r Lernende selbstständig verantwortlich. Damit jede/r Schüler/in die Aufgabenstellung des gestaltenden Vorlesens seiner/ihrer Leserolle in der jeweiligen Szene bearbeiten kann, sind verschiedene Phasen in wechselnden Sozialformen vorgesehen. Zu Beginn des Arbeitens in der Gruppe besitzt die Einzelarbeit einen hohen Stellenwert, denn wer nicht die Szene genau liest und nicht selbstständig die Bedeutung fremdsprachlicher Wendungen klärt, kann später in der Kooperation wenig dazu beitragen, wenn es darum geht, die Szene und die einzelnen Figurenrollen im Klassenverband mündlich vorzustellen. Danach steht das Üben des Vorlesens im Vordergrund. Um die Schüler/innen hierbei nicht zu überfordern, insbesondere aufgrund der Mehrsprachigkeit der Lesetheatertexte, ist im Lehr-Lern-Prozess der Einsatz verschiedener Trainingsformen vorgesehen (vgl. Teil C, Kap. 5). Das Besondere des Lesetrainings besteht darin, dass die Schüler/innen sich ihre Leserolle durch wiederholtes und begleitetes Lesen zu eigen machen, um sie dann lebendig vortragen zu können. Die zwei Hauptübungsformen, die im MELT-Projekt eingesetzt wurden, werden im Folgenden erläutert: (a) Tandemlesen und (b) Lesen mit Audiounterstützung.

(a) Die eingesetzte kooperative Lautlese-Methode des Tandemlesens basiert auf den beiden Grundprinzipien der Wiederholung und der Begleitung (vgl. Rosebrock/Nix 2014: 46f.). Beim Tandemlesen setzen sich die beiden Partner/innen nahe zueinander und legen das Skript ihrer Lesetheaterszene zwischen sich. Außerdem vereinbaren die Partner/innen, wer zuerst die Aufgabe des sogenannten „Trainers“ und wer die Aufgabe des „Sportlers“ übernimmt. Die Aufgabe des Trainers besteht darin, seine Leserolle, die er bereits geläufiger lesen kann als sein Partner, modellhaft so vorzulesen, dass der Sportler mitlesen und sein Lesen daran orientieren kann. Stockt beispielsweise der Sportler beim Lesen, passt der Trainer sein Lesetempo an und verlangsamt es. Unterläuft dem Sportler beim Lesen ein Fehler, korrigiert er sich selbst - sofern er es bemerkt - oder der Trainer korrigiert den Lesefehler und setzt wieder neu an, entweder am Anfang des Satzes oder eines Sinnabschnitts. Um das synchrone Lesen des Tandems zu koordinieren und die Aufmerksamkeit zu fokussieren, führt der Trainer beim Lesen den Finger mit. Auf diese Weise kann der Sportler mitlesen und sich am Lesemodell des anderen orientieren.

Bei dieser Form des Lautlesetrainings werden beide Leserollen mehrmals abwechselnd gelesen, sodass sich aufgrund wachsender Vertrautheit mit dem Text der Sichtwortschatz vergrößert, die Worterkennung automatisiert abläuft und sich die Lesegeschwindigkeit erhöht. Das gemeinsame Lesen mit einem/einer Lesepartner/in ermöglicht, gutes Vorlesen modellhaft zu erleben und nachzuahmen. Lesen beide Schü- 
ler/innen auf Wort- und Satzebene geläufiger, stehen ihnen mehr mentale Ressourcen zur Verfügung, um beim anschließenden gemeinsamen Üben der Gruppe auf die prosodischen Aspekte des Lesens zu achten.

Dieses Vorgehen ist sowohl angelehnt an die Methode des Paired reading von Topping (1989, 1995; zitiert nach Rosebrock 2014: 48f.) als auch an seine methodische Adaptation von Rosebrock/Nix (2014: 49f.) mit dem Titel Lautlese-Tandems. Im Gegensatz zu diesen beiden Verfahren ist aber beim Tandemlesen für das MELT-Projekt nicht das grundsätzliche Leistungsniveau im Bereich der Leseflüssigkeit ausschlaggebend bei der Aufgabenverteilung, sondern aufgrund der Spezialisierung auf die eigene Rolle ist der/ die Geübtere der Trainer und der/die weniger Geübtere ist der Sportler.

(b) Beim Lesen mit Audiounterstützung erhalten die Schüler/innen einen vorbereiteten Audiofile, auf dem sich Passagen in der Fremd- bzw. Migrationssprache der Lesetheaterskripts befinden. Die Aufgabe für die Lernenden besteht darin, über Kopfhörer die Stimme des Vorlesers bzw. der Vorleserin auf den Audiofiles anzuhören, dabei halblaut individuell mitzulesen und deren Aussprache gut zu imitieren und sicher bei der korrekten Aussprache zu werden. Das auf diese Weise begleitete Lautlesen eröffnet den Lernenden somit die Chance, das korrekte Vorlesen der oftmals herausfordernden fremd- und migrationssprachlichen Textpassagen anhand eines Modells zu erfahren. Ziel dieses Mitleseverfahrens ist es zudem, den Lesefluss der Lernenden so zu festigen, dass nach intensiven Übungsphasen die Unterstützung durch die Aufnahme bzw. durch die Lehrperson wegfallen kann und schlussendlich die Lernenden in der Lage sind, ohne fremde Hilfe diese Passagen sinngestaltend, korrekt und flüssig vorzulesen. Ein weiterer Vorteil ist, dass die Schüler/innen weitestgehend selbstständig zu einer korrekten Aussprache der herausfordernden Passagen in der Fremd- bzw. Migrationssprache gelangen.

(3) Hilfreiche Face-to-Face-Interaktion: Wie beim herkömmlichen Theater hängt der Erfolg einer mehrsprachigen Lesetheateraufführung wesentlich davon ab, wie gut die Interaktion zwischen den Akteur/innen und die Interaktion der Gruppe als Ganzes mit dem Publikum funktioniert. Kommunizieren und interagieren die Gruppenmitglieder nicht kooperativ, gelingt es wohl kaum, Nähe zum Publikum aufzubauen. Nur im Zusammenwirken aller Gruppenmitglieder wird die erzählte Geschichte lebendig und der berühmte Funke springt über. Damit das Zusammenspiel der Gruppe gelingt (z. B. richtiger Einsatz, Blickkontakt innerhalb der Gruppe), ist es wichtig, dass sich die Mitglieder gegenseitig anleiten, ermutigen und unterstützen. Während der Erarbeitung einer Szene tauschen sich die Lernenden unmittelbar darüber aus, wie ihr Vorlesen wirkt, ob der Einsatz der hör- und sichtbaren Mittel der Textgestaltung angemessen ist oder ob die schul- und fremdsprachlichen Passagen korrekt gelesen werden. Insbesondere während der Generalprobe interagieren die Schüler/innen einer Arbeitsgruppe intensiv in einer Atmosphäre unterstützender Interaktionen, indem sie Ideen für den Einsatz von Gestik und Mimik einbringen und/oder dabei etwas vorzeigen, das von den anderen imitiert werden kann. Die einzelnen Ideen der Gruppe werden auf ihre mögliche Wirkung für das Publikum miteinander reflektiert, dann wird entschieden, sie entweder umzusetzen oder als unpassend zu verwerfen. Alle Schüler/innen haben zwei unterschiedliche Rollen in Bezug auf das Lernen im Mehrsprachigen Lesetheater, die an verschiedene Funktionen geknüpft sind: Zum einen sind sie selbst Akteur/innen auf 
der Bühne, zum anderen aber auch Teil des zuhörenden und zuschauenden Publikums. Jede Aufführung der Schülergruppen gibt daher aufgrund der Beobachtungen, wie andere Gruppen die Aufgabenstellung lösen, Impulse für weitere Durchführungen.

(4) Feedback: Im Lehr-Lern-Prozess sind zwei Schritte mit expliziten Phasen vorgesehen, in denen die Schüler/innen Rückmeldungen von anderen einholen oder selber solche geben: Dies geschieht einmal im Zusammenhang des halblauten Lesens und Übens in Einzel- und Partnerarbeit, das andere Mal während der Generalprobe zur Vorlesegestaltung (vgl. Teil C, Kap. 5). Darüber hinaus haben die Schüler/innen vielfältige Gelegenheiten sich gegenseitig unterstützendes Feedback zu geben, beispielsweise bei der Klärung von Meinungsverschiedenheiten oder beim Austausch über effektive Lerntechniken. Die Rückmeldungen der Lehrperson während der Generalprobe und die Reaktionen des zuhörenden Publikums während der Aufführung geben Hinweise darauf, wie erfolgreich die Gruppe zusammengearbeitet hat. Der Applaus am Ende des Auftritts ist wie im richtigen Theater ein „Feedback“ für die gezeigte Leistung und Ausdruck des Gefallens des Publikums.

(5) Angemessene Nutzung kooperativer Fertigkeiten: Das gemeinsame Lernen und Arbeiten in unterstützender Interaktion erfordert von den Schüler/innen „interpersonal and small group skills" (Johnson/Johnson 1999: 82). Um die Zielsetzungen des kooperativen Unterrichts zu erreichen, müssen die Lernenden nach Johnson/Johnson (1987: 109ff., zitiert nach Borsch 2015: 31ff.) besondere Fähigkeiten der Kommunikation (z. B. Empathiefähigkeit, klare, eindeutige und vollständige Kommunikation), die Fähigkeit zum Aufbau eines Vertrauensklimas (z. B. Vertrauensbildung durch gegenseitige Akzeptanz und Unterstützung, wertschätzende Behandlung der Beiträge der einzelnen Gruppenmitglieder), die Bereitschaft zur Übernahme von Gruppenführungsaufgaben (z. B. Diagnose und Feststellung der notwendigen Arbeitsschritte, zielführende Bereitstellung und Verwendung der Ressourcen unterschiedlicher Gruppenmitglieder) und Kompetenzen zum Umgang mit Kontroversen erwerben (z. B. gemeinsames Finden von Lösungsansätzen, keine Äußerung personenbezogener Kritik). Bei der kooperativen Fertigkeit des Konfliktmanagements spielen bei unterschiedlichen Interessen und Neigungen zudem auch Strategien der Entscheidungsfindung eine Rolle, die die Lernenden bei MELT angemessen nutzen müssen. Bei MELT werden die Schüler/innen im Verlauf des Lehr-Lern-Prozesses in den oben genannten kooperativen und sozialen Fertigkeiten gefördert (vgl. Tab. 1 in folgendem Abschnitt 3.2). Die Lehrperson sollte also auf Bedingungen achten, die soziales Verhalten ermöglichen; sie organisiert die Gruppen so, dass z. B. arbeitsteilig unterschiedliche Rollen eingenommen werden, etwa für die Moderation des Arbeitsprozesses oder das Zeitmanagement der Gruppe. Bei Konflikten in der Gruppe, die den Lernprozess unangemessen lange blockieren (z. B. wer welche Rolle lesen darf, Störungen durch die fehlende Beteiligung Einzelner), interveniert die Lehrperson bei Bedarf und moderiert mit der Gruppe Konfliktlösungsmöglichkeiten.

(6) Konstruktive Unterstützung und Reflexion der Gruppenprozesse: Obwohl im LehrLern-Prozess kein eigener separater Schritt vorgesehen ist, in dem die Gruppe ihren Arbeitsprozess überdenkt, sollten regelmäßig Phasen eingeschaltet werden, in denen die Gruppen die Gelegenheit erhalten, ihre Zusammenarbeit und ihr gemeinsames Lernen zu reflektieren. Es können für solche Phasen unterschiedliche Schwerpunkte 
gesetzt werden, die sich auf methodische, soziale oder inhaltliche Aspekte des Lernens mit dem Mehrsprachigen Lesetheater beziehen. Für Reflexionsphasen eignen sich die folgenden drei Zeitpunkte im Ablauf besonders, in jeder Phase kann ein anderer Aspekt fokussiert werden: (a) Im Anschluss an die intensive Übungsphase der Leserollen durch wiederholtes halblautes Lesen ist es sinnvoll, wenn die eingesetzte Übungsstrategie Gegenstand der Reflexion ist (vgl. Abb. 1). (b) Nach der Generalprobe, während der die Gruppe nochmals ihren Auftritt abstimmt, empfiehlt sich die Reflexion sozialer Aspekte, beispielsweise wie gut die Gruppe bisher zusammenarbeitet hat, ob und wie sich die einzelnen Mitglieder unterstützt haben, welche Schritte oder Maßnahmen bisher gut bzw. weniger gut funktioniert haben und warum etc. (vgl. Abb. 2). (c) Nach der den Lernprozess abschließenden Aufführung des Lesetheaters kann der Auftritt selbst fokussiert werden. Relevante Reflexionsaspekte sind z. B. die persönliche Zufriedenheit mit der Qualität der Aufführung, die Einschätzung der Wirkung auf das Publikum, aber auch der gesamte Gruppenprozess während der Durchführung. Wird nicht nur das Gruppenprodukt, also die Aufführung, sondern auch der Gruppenprozess zum Thema, achten die Schüler/innen stärker auf das Verhalten in der Gruppe, z. B. werden „Anstrengungserfahrungen“ (Borsch 2015: 128) begünstigt. Damit die Schüler/innen in konstruktiver Weise sich über ihre Zusammenarbeit austauschen, empfiehlt es sich, Leitfragen vorzugeben, zu denen sich alle offen äußern dürfen und die das erwünschte Positive im Zusammenarbeiten in den Vordergrund rücken.

\begin{tabular}{|l|l|c|c|c|c|c|l|}
\hline \multicolumn{2}{|l|}{ Beim Arbeiten im Tandem... } \\
\hline 1. & $\begin{array}{l}\text { haben wir die Zeit gut zum } \\
\text { Lernen genutzt. }\end{array}$ & ++ & + & 0 & - & -- & $\begin{array}{l}\text { haben wir die Zeit zum Lernen } \\
\text { nicht so gut genutzt. }\end{array}$ \\
\hline 2. & $\begin{array}{l}\text { haben wir uns gegenseitig } \\
\text { geholfen. }\end{array}$ & ++ & + & 0 & - & -- & $\begin{array}{l}\text { haben wir uns gegenseitig nicht } \\
\text { so gut geholfen. }\end{array}$ \\
\hline 3. & $\begin{array}{l}\text { haben wir uns schwierige } \\
\text { Wörter erklärt. }\end{array}$ & ++ & + & 0 & - & -- & $\begin{array}{l}\text { haben wir uns schwierige Wörter } \\
\text { nicht erklärt. }\end{array}$ \\
\hline
\end{tabular}

Ein Verbesserungsvorschlag:

Ein Lob, was besonders gut gelungen ist:

Abbildung 1: Unsere Arbeit im Tandem (Quelle: Sabine Kutzelmann/Andrea Büchel 2016, unveröffentlichter Feedbackbogen, Rorschach)

\begin{tabular}{|l|l|c|c|c|c|c|l|}
\hline Beim & Arbeiten in der Gruppe... \\
\hline 1. & $\begin{array}{l}\text { haben alle Verantwortung } \\
\text { für das Lesetheater über- } \\
\text { nommen. }\end{array}$ & ++ & + & 0 & - & -- & $\begin{array}{l}\text { haben einige keine Verant- } \\
\text { wortung für das Lesetheater } \\
\text { übernehmen wollen. }\end{array}$ \\
\hline 2. & $\begin{array}{l}\text { haben wir von allen in der } \\
\text { Gruppe die Ideen für die } \\
\text { Aufführung miteinander } \\
\text { besprochen. }\end{array}$ & ++ & + & 0 & - & -- & $\begin{array}{l}\text { wollten Einzelne ihre Ideen } \\
\text { durchsetzen, obwohl die } \\
\text { anderen in der Gruppe nicht } \\
\text { einverstanden waren. }\end{array}$ \\
\hline
\end{tabular}




\begin{tabular}{|l|l|l|l|l|l|l|l|}
\hline 3. & $\begin{array}{l}\text { haben wir uns gegensei- } \\
\text { tig unterstützt und gute } \\
\text { Tipps zum Lesen der Rollen } \\
\text { gegeben. }\end{array}$ & ++ & + & 0 & - & -- & $\begin{array}{l}\text { haben einige keine Tipps und } \\
\text { Unterstützung zum Lesen der } \\
\text { Rollen gegeben. }\end{array}$ \\
\hline 4. & $\begin{array}{l}\text { haben wir uns bemüht, die } \\
\text { fremdsprachlichen Textstel- } \\
\text { len zu verstehen und für die } \\
\text { Zuhörenden verständlich } \\
\text { vorzulesen. }\end{array}$ & ++ & + & 0 & - & -- & $\begin{array}{l}\text { haben wir uns wenig bemüht, } \\
\text { die fremdsprachlichen Text- } \\
\text { stellen zu verstehen und für } \\
\text { die Zuhörenden verständlich } \\
\text { vorzulesen. }\end{array}$ \\
\hline
\end{tabular}

Unterschrift Moderator/in:

Abbildung 2: Unsere Arbeit in der Gruppe (Quelle: Sabine Kutzelmann/Andrea Büchel 2016, unveröffentlichter Feedbackbogen)

Die Schüler/innen müssen in den Prozess des kooperativen Arbeitens nach und nach hineinwachsen. Soll kooperatives Lernen gelingen, setzt dies einerseits bei den Lernenden bestimmte grundlegende Kompetenzen voraus. Dazu zählen beispielsweise die Bereitschaft der Schüler/innen, sich auf einen gemeinsamen, oft nicht immer einfachen Prozess der Auseinandersetzung rund um das mehrsprachige Lesetheaterstück einzulassen, sich anderen mitzuteilen oder den/die andere/n in jeder Phase des Lehr-und Lernprozesses wertzuschätzen. Andererseits ändert sich beim kooperativen Arbeiten auch die Rolle der Lehrperson inklusive ihrer Aufgaben; im Folgenden wird darauf eingegangen, inwiefern dies zutrifft.

\subsection{Rolle und Aufgaben der Lehrperson}

Der Lehrkraft wird als unterrichtsgestaltende Größe eine wichtige Rolle zuteil; sie plant und gestaltet den Unterricht und ist dafür verantwortlich, förderliche Bedingungen des kooperativen Lernens zu schaffen. Zu Beginn der Implementierung der Fördermethode Mehrsprachiges Lesetheater besteht die zentrale Aufgabe der Lehrperson zunächst darin, den Schüler/innen zu erläutern, was sie bei der Bearbeitung der Aufgabenstellung lernen sollen, was die zentralen Schritte bis zur Aufführung eines mehrsprachigen Lesetheaterstücks sind und welche Aktivitäten jeweils anstehen. Der sorgfältigen Einführung durch die Lehrkraft kommt eine hohe Bedeutung zu, es geht nämlich darum, dass die Schüler/innen nach zwei bis drei Durchgängen fähig sind, ohne oder nur noch mit geringer Unterstützung die einzelnen Schritte des kooperativen Lernens selbstständig in ihren Arbeitsgruppen auszuführen.

Beim kooperativen Lernen mit MELT werden neben fachlichen immer auch soziale und kooperative Zielsetzungen verfolgt, darum sollte die Lehrperson bei der Einführung der einzelnen Phasen des kooperativen Lernens in den Unterricht diese Zieldimensionen thematisieren sowie den genauen Ablauf bei der Bearbeitung der Teilaufgaben darstellen. Hinsichtlich der sozialen Zielsetzungen ist wichtig, den Schüler/innen zu verdeutlichen, dass für eine erfolgreiche Aufführung einer mehrsprachigen Lesetheaterszene das Zusammenspiel der Gruppe (z. B. richtiger Einsatz, Blickkontakt zur Grup- 
pe) ebenso wichtig ist wie die individuelle Leistung des/der Einzelnen; zumal sowohl der/die Einzelne als auch die Gruppe als Ganzes Mut zum Auftreten vor Publikum benötigen. Eine Herausforderung für jede/n Einzelne/n sowie für die Gruppe ist hierbei zu lernen, auch mit Mitschüler/innen fachlich zusammenzuarbeiten, mit denen man eigentlich nicht so gut zurechtkommt oder gegen die man eventuell persönliche Antipathien empfindet.

In Tabelle 1 werden die spezifischen Zielsetzungen in den verschiedenen Phasen des kooperativen Lernens dargestellt.

Tabelle 1: Übersicht über die fachlichen, sozialen und kooperativen Zielsetzungen des kooperativen Lernens

\section{Zielsetzungen}

Fachliche Fertigkeiten

Lesen der Lesetheaterszene und Rollenverteilung

- Klären zentraler Inhalte für das Verständnis der Lesetheaterszene

- Notiztechniken kennen und anwenden

- Hilfsmittel (z. B. Übersetzungen, Audiofiles) gezielt einsetzen
}

Soziale und kooperative Fertigkeiten
- Individuell gewonnenes Verständnis wie auch Lern- und Arbeitstechniken einbringen und weitergeben (Vertrauensbildung)

- unterschiedliche Interessen und Neigungen bei der Rollenverteilung berücksichtigen (Strategien der Entscheidungsfindung)

Erarbeiten einer Inhaltsangabe der Lesetheaterszene

- Klären des Inhalts schwieriger Sätze und/ oder die Bedeutung von Schlüsselwörtern

- das Wichtigste zusammenfassen

- Notiztechniken kennen und anwenden Vorstellen der Szenen und der Figurenrollen

- Wiedergeben des Handlungszusammenhangs Üben der Leserollen und gegenseitiges Feedback

- Lesegenauigkeit und Automatisierung in den ersten Übungsdurchgängen

- Sicherheit bei der prosodischen Vorlesegestaltung

- Feedbackmethoden kennen und anwenden
- Verantwortung für die gemeinsame Verständnissicherung übernehmen

- gegenseitige Unterstützung bei Schwierigkeiten (Empathiefähigkeit)
- mit anderen zusammen Kurzpräsentationen erarbeiten

- miteinander und/oder alleine die Szene in Form einer Kurzpräsentation vorstellen (angemessenes Führungsverhalten)
- sich an die vereinbarte Übungsstrategie halten

- Feedback einerseits sachlich und angemessen geben und andererseits auch annehmen können 
Generalprobe und Feedback

- Aufeinander abstimmen der einzelnen Leserollen

- Planung des Auftritts (Bühnenpositionen, Einsatz von Notenständern etc.)

- Wirkung der Vorlesegestaltung reflektieren

- Feedbackmethoden kennen und anwenden

- Ideen von anderen wahrnehmen und wertschätzen (Empathiefähigkeit)

- Feedback einerseits sachlich und angemessen geben und andererseits auch annehmen können (Strategien der Entscheidungsfindung)

\section{Aufführung}

- Zeigen der Lernfortschritte im Bereich des gestaltenden Vorlesens (Leseflüssigkeit)

- Erhöhung der Sicherheit beim Präsentieren (Auftrittskompetenz)
- Mitverantwortung für die Vorlese-Inszenierung tragen

- ermutigend und solidarisch handeln (Führungsverhalten, Empathiefähigkeit)

Ein kompletter Durchgang aller acht Schritte des gesamten Lehr-Lern-Prozesses erfolgt in der Regel innerhalb einer Unterrichtswoche (ca. drei bis fünf Unterrichtsstunden). Der Ablauf des kooperativen Lernens in der Wochenplanung lässt sich z. B. auf einem Plakat darstellen (vgl. Tab. 1, die grau hinterlegten Titel der Einzelschritte) und sollte nach zwei bis drei Durchführungen des gesamten Prozesses verinnerlicht werden. Sobald Routinen in Bezug auf die Folge der Einzelschritte aufgebaut sind, dient die Visualisierung den Untergruppen zur selbstständigen Orientierung beim Umgang mit neuen mehrsprachigen Lesetheatern. Ab dann besteht die Hauptaufgabe der Lehrperson darin, den Prozess zu koordinieren und die Arbeitsgruppen zu begleiten. Das Begleitverhalten der Lehrperson wird im Folgenden kurz skizziert.

Auch wenn die Lehrperson während der Phasen der schülerzentrierten Interaktion in den Hintergrund tritt, da der wechselseitige Bezug im Lernprozess zwischen den Schüler/innen einer Arbeitsgruppe stattfindet, begleitet die Lehrperson intensiv den Lernprozess ihrer Schüler/innen und unterstützt die Gruppen. Vor allem für die folgenden Aspekte ist es wichtig, dass die Lehrperson, die Gruppen mit gezieltem „Eingreifen“ begleitet: (a) Kompetenzaufbau im gestaltenden Vorlesen, (b) Interaktions- und Durchnahmetempo, (c) Aufbau von Übungsmotivation und (d) Übernahme von Aufgaben als Moderator/in.

(a) Kompetenzaufbau im gestaltenden Vorlesen: Beim Vorlesen eines Textes wird das eigene Textverständnis deutlich gemacht und der Text mithilfe der eigenen Stimme und des Körpers gestaltet. Während des Gestaltungsprozesses suchen Vorlesende nach Ausdrucksmöglichkeiten und experimentieren mit den ihnen zur Verfügung stehenden Mitteln der Stimme und des Körpers. Um die Angemessenheit der Gestaltung einschätzen zu lernen, benötigen Lernende unmittelbare Reaktionen von anderen, die ihnen Orientierung geben, inwieweit eine Gestaltung überzeugen kann, sie gelungen erscheint und vielleicht sogar mitreißen kann etc. Neben den Peers soll und darf der/die Lehrer/in den Gestaltungsprozess beeinflussen durch seine/ihre Rückmeldungen, die auf ihren Wahrnehmungen als Zuschauer/in und Zuhörer/in beruhen. Zudem unterstützt die Lehrperson auch besonders die Lernenden mit fremdsprachlichen Rollen bei der Aussprache und bei der Gestaltung der entsprechenden Textstellen; sie steht jeder Gruppe für Fragen sowie Feedbacks zur Verfügung. Während der Phasen, in denen entweder 
in Partner- oder Gruppenarbeit die Figuren- und Erzählerrollen laut gelesen werden, sind zusätzliche Arbeitsräume vorteilhaft. Damit die Lehrperson auf Fragen der Schüler/innen dennoch zeitnah eingehen kann, um etwa bei der Aussprache fremdsprachlicher Wendungen zu helfen, sollten diese Übungsräume in der Nähe des Klassenzimmers liegen.

(b) Interaktions- und Durchnahmetempo: Idealerweise haben die Schüler/innen die Zeit zur Verfügung, die sie für ihren Lernprozess zur Bearbeitung der einzelnen Teilaufgaben benötigen. Da erfahrungsgemäß manche Gruppen „oberflächlicher“ als andere arbeiten, gehört es zur Aufgabe der Lehrperson, diese Gruppen konstruktiv-kritisch dabei zu unterstützen, ein angemessenes Interaktionstempo (vgl. Borsch 2015: 46) zu finden und die zur Verfügung stehende wertvolle Unterrichtszeit produktiv zu nutzen. Dabei hängt die Interaktionsqualität natürlich von den kooperativen bzw. kommunikativen Fertigkeiten der Schüler/innen ab (vgl. ebd.: 47). Es ist daher hilfreich, diese Fertigkeiten kontinuierlich zu trainieren. Dabei verlangt die Lehrperson Leistung und bewertet Anstrengungen positiv. Etwas herausfordernder beim kooperativen Arbeiten in MELT sind die Anpassung des Unterrichtstempos und die Aufgabenschwierigkeiten der einzelnen Lehr- und Lernprozesse an die heterogenen Lerndispositionen der Schüler/ innen. Hierbei ist eine sehr gute Einschätzung der einzelnen Lerngruppen bzw. Schüler/innen um die benötigte Zeit für die jeweiligen Aufgaben von Vorteil. So kann die Lehrperson bereits bei der Planung die unterschiedlichen Durchnahmetempi berücksichtigen und geeignete Beschäftigungen sowie eventuell Zusatzaufgaben bereithalten (vgl. ebd.: 47).

(c) Aufbau von Übungsmotivation: Um bei den Lernenden die Motivation zum Üben der Lesepassagen aufzubauen, müssen schülergerechte Erklärungen zum Sinn und der Notwendigkeit von Lautlese-Übungen (Tandemlesen, Lesen mit Audiounterstützung) gegeben werden, damit die Schüler/innen die Relevanz der zugrunde liegenden Prinzipien der Wiederholung und der Begleitung verstehen. Des Weiteren muss vor allem zu Beginn der Arbeit mit einem mehrsprachigen Lesetheaterstück die Dringlichkeit aufgezeigt werden, gegebenenfalls auch anspruchsvolle kleinere Teilstücke (z. B. fremdsprachliche Passagen) isoliert und wiederholend zu üben. Ein Bezug zum Thema Musik mit dem Hinweis, dass Musiker/innen zum Einüben eines Musikstückes auch nicht immer das ganze Werk vom Anfang bis zum Ende durchspielen, sondern vielmehr kleine, schwierige Teilstücke wiederholt isoliert einüben, mag ebenfalls hilfreich sein.

(d) Übernahme von Aufgaben als Moderator/in: Zu den Aufgaben der Lehrpersonen gehört auch, verschiedene (Leitungs-)Aufgaben an bestimmte Schüler/innen zu übertragen und sie bei der Erfüllung dieser Aufgaben zu unterstützen. Die Lernenden werden dadurch mit der Funktion eines Moderators/einer Moderatorin beauftragt. Dies kann laut Borsch (2015: 138) in zwei Schritten erfolgen: Zunächst wird erarbeitet, welche Aspekte für die Leitung einer Gruppe wichtig sind (z. B. durch ein Brainstorming), danach kann in Rollenspielen bzw. im Modeling der Lehrperson der Umgang mit bestimmten herausfordernden Situationen geübt werden (z. B. Situationen, die ein gewisses Konfliktpotenzial beinhalten). Es könnten beispielweise folgende Aufgaben an Moderator/innen delegiert werden: Zeitwächter, Rollenverteilung, Beteiligung aller Gruppenmitglieder an den verschiedenen Prozessschritten, keine Dominanz einzel- 
ner Mitschüler/innen, Feedback geben, Feststellung der nötigen Arbeitsschritte etc. Die Lesetheatertexte wurden für das Projekt MELT so konzipiert, dass sie sich auf eine Klassengröße von bis zu 25 Schüler/innen hin leicht adaptieren lassen. Mehrsprachiges Lesetheater kann aber auch in größeren Klassen durchgeführt werden; hierfür müssen aber entsprechende Anpassungen bei der Aufteilung der Gruppen und der Organisation vorgenommen werden. Andere Schülerzahlen können durch eine Zusammenlegung von Leserollen ausgeglichen werden. In dieser Situation ist es wichtig, dass die Lehrperson die Rolle des Moderators/der Moderatorin übernimmt.

Neben der besonderen Rolle der Lehrperson bei der Einführung des Mehrsprachigen Lesetheaters und spezifischer Verhaltensweisen beim Begleiten der Gruppen gehört auch die Beurteilung von Schülerleistungen zu ihren Aufgaben. Es ist durchaus möglich, den einzelnen Schüler/innen einer Arbeitsgruppe Noten für ihre jeweils erbrachten Aufführungsleistungen anhand der vorher vereinbarten Kriterien des gestaltenden Vorlesens zu geben. Im Vordergrund sollte aber unseres Erachtens die formative Beurteilung stehen. Es kann sowohl die Aufführung der Lesetheaterszene, also das Produkt der Gruppenarbeit, als auch der Prozess der Erarbeitung einer Szene beurteilt werden. Bei einer solchen Beurteilung würde das individuelle Arbeitsverhalten der Schüler/innen während des kooperativen Lernens im Vordergrund stehen (vgl. die sozialen und kooperativen Fertigkeiten in Tab. 1).

\subsection{Ausblick: Förderung sozialer und kommunikativer Kompetenzen}

Obwohl beim Lernen mit dem Mehrsprachigen Lesetheater der Lernfokus auf der Förderung der Leseflüssigkeit und der Lesemotivation liegt, können mit der Strukturierung des Designs durch Elemente kooperativen Lernens auch soziale, kooperative und kommunikative Kompetenzen unterstützt werden. Werden diese durch die bereits erwähnten sozialen und kooperativen Zielsetzungen erreicht, kann diese Lesefördermethode zu einem guten und anregenden sozialen Klima in der Klasse beitragen. Empirische Forschungsergebnisse lassen vermuten, dass diese Kompetenzen und Lernziele durch kooperatives Lernen gefördert werden können. Im Folgenden werden einige Wirkungen hinsichtlich sozialer und kommunikativer Kompetenzen kurz vorgestellt.

Borsch (2015: 126) weist auf positive Effekte kooperativen Lernens in Bezug auf emotionale und soziale Lernziele hin. Er belegt dies anhand einer detaillierten Zusammenstellung einiger Studien von Slavin (1995) zu den folgenden Themenfeldern: Beziehungen zwischen den Schüler/innen, Hilfsbereitschaft, motivationale Überzeugungen, Lernfreude, Aufmerksamkeit, kooperative Zusammenarbeit und Selbstwertgefühl. Die Wirksamkeit kooperativer Lernformen wurde zudem in Metaanalysen international empirisch untersucht (z. B. Slavin 1995; Rohrbeck et al. 2003; Ginsburg-Block/ Rohrbeck/Fantuzzo 2006). Insgesamt belegen sie die überwiegend positive Wirkung kooperativen Lernens besonders auf motivationale, soziale (Zugewinn an sozialen Fähigkeiten, Verbesserung kooperativer Verhaltensweisen) und emotionale (verbessertes Selbstkonzept) Lernziele:

In summary, cooperative learning has been shown in a wide variety of studies to positively influence a host of important noncognitive variables. Although not every study 
has found positive effects on every noncognitive outcome, the overall effects of cooperative learning on student self-esteem, peer support for achievement, internal locus of control, time on-task, liking of class and of classmates, cooperativeness, and other variables are positive and robust (Slavin 1995: 70).

Des Weiteren belegen Johnson/Johnson (1994: 37, zitiert nach Bonnet/Küppers 2011: 38) aus lernpsychologischer Sicht eine stärkere Unterstützung der Lernenden, eine Reduzierung der Angst zum freien Sprechen, die Zunahme gegenseitigen Vertrauens und eine Erhöhung der positiven Einstellung gegenüber Andersartigkeit. Die Zunahme sozialer Kompetenzen auf der individuellen Ebene ist ebenfalls nachgewiesen (vgl. Jurkowski/Hänze 2008, zitiert in Bonnet/Küppers 2011: 38).

Zudem gelingt kooperatives Lernen nach dem aktuellen Forschungsstand vor allem dann, wenn neben den Lernergebnissen auch Lernprozesse Beachtung finden (vgl. Traub 2004). Dies ist gerade im MELT-Design der Fall. Theoretische Überlegungen und empirische Befunde lassen zudem vermuten, dass die Kombination kooperativen Lernens mit einer Feedback-Maßnahme bei geeigneter Umsetzung zu einer positiven Einschätzung der eigenen Leistung führen kann, was wiederum zum Weiterlernen motivieren kann (vgl. z. B. Krause 2007: 189). Haag, Fürst und Dann (2000) dokumentieren darüber hinaus in ihrer Studie zum erfolgreichen Gruppenunterricht, dass präzise und eindeutige Arbeitsaufträge erheblich zur Orientierung der Lernenden beitragen. Dadurch werden Interventionen durch die Lehrperson vermeidbar. Huber, Konrad und Wahl (2001) untersuchten spezielle kooperative Lernmethoden und stellten fest, dass die Versuchsgruppen bessere Ergebnisse in sozialen Verhaltensweisen zeigten und zudem deren Motivation im Vergleich zu den Mitschüler/innen der Kontrollgruppen höher war.

Bei den Lernmaterialien handelt es sich um mehrsprachige Lesetheaterstücke. In Hinblick auf den Fremdsprachenerwerb ist vor allem die Steigerung der Sprechantei$l e$ in der jeweiligen schulisch geförderten Fremdsprache (je nach Anwendungskontext vorwiegend Englisch oder Französisch) sowie der Wortschatzerwerb von Bedeutung (vgl. auch z. B. McDonell 1992, zitiert in Bonnet/Küppers 2011: 38; Theinert 2013). Des Weiteren konnten Studien zum kooperativen Lernen im Fremdsprachenunterricht zeigen, dass bei Schüler/innen in den Versuchsgruppen Sprachhemmungen abgebaut werden, die Lernenden die Fremdsprache flüssiger verwenden, insgesamt mehr Strategien bei der mündlichen Kommunikation eingesetzt werden und bei den Lernenden das Selbstwirksamkeitserleben erhöht wird (vgl. Theinert 2013). Kooperative Lernformen wirken sich also neben den genannten sozialen Lernzielen auch bedeutend auf die mündliche Kommunikationsfähigkeit der Schüler/innen aus. Es spricht viel dafür, dass die im Mehrsprachigen Lesetheater auch angestrebten sozialen und kommunikativen Lernziele durch kooperatives Lernen gefördert werden können.

Eine Geschichte in zwei oder mehr Sprachen auf einer Bühne vor Publikum zum Leben zu erwecken, darin besteht die anforderungsreiche Aufgabe für Kinder und Jugendliche bei der Aufführung eines mehrsprachigen Lesetheaterstücks. Wie in diesem Kapitel dargelegt, soll die Konzeption des kooperativen Lernens der Lesefördermethode Mehrsprachiges Lesetheater dazu beitragen, dass alle Schüler/innen sich hierbei im wechselseitigen Miteinander als Gruppe und Klasse als „Stars“ erleben können, denen gern zugehört und zugeschaut wird, wie sie Figuren und Charaktere lebendig inszenieren (vgl. Martinez/Roser/Strecker 1998/1999). 